\title{
Shear zones between rock units with no relative movement
}

\author{
H. Koyi (1), H. Schmeling (2), S. Burchardt (3), C. J. Talbot (4), S. Mukherkjee (5), H. Sjöström (6), and Z. \\ Chemia (7) \\ (1) Department of Earth Sciences, Uppsala University, Uppsala, Sweden, hemin.koyi@ geo.uu.se, (2) Institute of Earth \\ Sciences, J. W. Goethe-University Frankfurt, Germany, schmeling@geophysik.uni-frankfurt.de, (3) Department of Earth \\ Sciences,, Uppsala University, Uppsala, Sweden, steffi.burchardt@geo.uu.se, (4) Department of Earth Sciences,, Uppsala \\ University, Uppsala, Sweden, Christopher.Talbot@geo.uu.se, (5) Department of Earth Sciences, Indian Institute of \\ Technology, Bombay, India, soumyajitm@gmail.com, (6) Department of Earth Sciences, Uppsala University, Uppsala, \\ Sweden, Hakan.Sjostrom@geo.uu.se, (7) Geological Survey of Norway (NGU), Trondheim, Norway, zch@geo.ku.dk
}

Shear zones are normally viewed as relatively narrow deformation zones that accommodate relative displacement between two "blocks" that have moved past each other in opposite directions. This study reports localized zones of shear between adjacent blocks that have not moved past each other. Such zones, which we call wakes in this study, form due to the movement of exotic blocks within a viscous medium (denser blocks sinking within a salt structure, boudin tracks), melt in a partially molten surroundings (melt movement during migmatisation), or solid blocks sinking through a partially molten magma body (stoping). From the fluid dynamics point of view these shear zones can be regarded as the low Reynolds number deformation zones within the wake of a body moving through a viscous medium. While compact (aspect ratio 1:1:1) moving bodies generate axial symmetric (cone like) shear zones, elongated bodies (vertical plates or horizontal rod-like bodies) produce tabular shear zones. Unlike conventional shear zones across which shear indicators ideally display consistent symmetries, shear indicators on either sides of the shear zone reported here show reverse kinematics. Thus profiles exhibit a combination of sinistral and dextral shear zones across their center-lines or -planes.

We have used field observations and model results to suggest that examples of transit paths develop where denser blocks sink within salt structures, bodies of melt rise through migmatites, between boudins separated by progressive extension and (perhaps) where slabs of subducted oceanic lithosphere delaminate from the continental crust and sink into the asthenosphere. We also argue that such shear zones may be more common than they have been given the credit for and may be responsible for some of the kinematic reversals reported in shear zones. 